\title{
Study on the Fracture Evolution Law of Coal Based on Image Retrieval Method and X-Ray Computed Tomography
}

\author{
Yan Wu $\mathbb{D}^{1,2}$ Dengke Wang $\mathbb{D}^{3,4}{ }^{3,4}$ Xiaofei Pang, ${ }^{3}$ and Hang Zhang ${ }^{3}$ \\ ${ }^{1}$ Institute of Deep Earth Sciences and Green Energy, Shenzhen University, Shenzhen 518060, China \\ ${ }^{2}$ College of Civil and Transportation Engineering, Shenzhen University, Shenzhen 518060, China \\ ${ }^{3}$ State Key Laboratory Cultivation Base for Gas Geology and Gas Control, Henan Polytechnic University, Jiaozuo 454000, China \\ ${ }^{4}$ Collaborative Innovation Center of Coal Work Safety and Clean High-Efficiency Utilization, Jiaozuo 454000, China
}

Correspondence should be addressed to Dengke Wang; wdk@hpu.edu.cn

Received 17 January 2022; Revised 13 February 2022; Accepted 18 February 2022; Published 4 March 2022

Academic Editor: Peng Hou

Copyright (c) 2022 Yan Wu et al. This is an open access article distributed under the Creative Commons Attribution License, which permits unrestricted use, distribution, and reproduction in any medium, provided the original work is properly cited.

\begin{abstract}
The combination of real-time computed tomography (CT) scanning test and loading test can visualize the evolution process of defects such as internal pores and fissures in the coal. The axial deformation of the loaded coal sample will change the layers of the same section in the real-time CT scanning test, which will affect the analysis of fracture progression. To address the concern, we presented a CT image similarity retrieval method. The dynamic development law of fracture networks under triaxial stress was investigated using image processing technologies. The results reveal that the CT images of the coal sample in the same section can be obtained using the proposed CT image similarity retrieval method, which can more accurately characterize the fracture distribution and dynamic evolution law. The coal sample under triaxial stress experienced three fracture evolution stages: the closure in the compaction and elastic stages, the initiation and development in the plastic yield stage, and the rapid expansion and penetration in the postpeak stage. The variation trend of two-dimensional fracture ratio and fracture volume was consistent. The analysis is conducive to clarifying the coal failure mechanism under triaxial stress.
\end{abstract}

\section{Introduction}

The coal contains many holes, fissures, and other defects. Under engineering disturbance, the instability and failure of the coal body are promoted by the initiation, expansion, interaction, and penetration of defects [1-4]. The complex fracture structure impacts coal deformation and failure [5-8]. The nonuniform distribution and expansion evolution of fractures lead to variations in the permeability and mechanical characteristics of the coal body [9-13]. Therefore, studying and analyzing the dynamic development of fractures might give a theoretical foundation for understanding the coal failure process.

X-ray CT has the advantages of high accuracy, nondestructive testing, and the ability to visualize the interior structure of the sample. As a result, it has become increasingly popular in investigating mechanical properties and seepage characteristics of coal and rock materials in recent years [14-17]. Kai and Zheng [18] used acoustic emission
(AE) and X-ray CT to conduct real-time damage assessments on water-bearing sandstone. They discovered a mapping relationship between water content and damage degree. Zhao et al. [19] investigated the pore structure features of coal by using nano-CT equipment. They conducted computational fluid dynamics (CFD) simulations based on nanopores and discovered the permeability is anisotropic. Wang et al. $[14,16,20]$ reconstructed the internal threedimensional structure of coal and found that the permeability and porosity of coal are negatively correlated with the volume fractal dimension.

The macroscopic failure and instability of coal are the results of the evolution and development of pores and fissures. Many researchers have analyzed and discussed it in various degrees $[21,22]$. The coal matrix, minerals, and fractures in CT images were segmented and reconstructed, displaying the spatial distribution of their three parts in coal intuitively and stereoscopically and addressing the effects of minerals on fracture expansion $[23,24]$. Wang et al. 
[25] and Liu et al. [26] believed that the thermal stress exceeding the tensile strength of the coal sample is the direct reason for the initiation, propagation, and interconnection of cracks under temperature impact. In addition, Zhong et al. [27], Wang et al. [28], and Tan et al. [29] carried out a uniaxial compression test and real-time CT scanning tests on the coal and rock. They used CT images to analyze the fracture propagation and damage evolution process. During triaxial compression tests, Liu et al. [30] partitioned CT images of rock and defined the new damage variable, which can predict rock damage and failure. Combined with gray level cooccurrence matrix (GLCM) theory and X-ray CT, Wang et al. [31] analyzed gas-bearing coal's fracture dynamic evolution law under triaxial stress.

At present, when analyzing the dynamic evolution of the coal and rock fractures under external loading, most researchers can only select CT images of fixed layers for analysis [27-31]. Obviously, under external loading, the axial deformation of the coal and rock can alter the layers of the same section under different deformation states in the real-time CT scanning test, and there will be errors in analyzing the evolution of the internal mesostructure. Therefore, the CT images of the coal sample in the same section were obtained based on image retrieval technology and made qualitative and quantitative analyses and more accurately characterize the fracture dynamic evolution law of coal in the loading process.

\section{CT Image Retrieval Method}

2.1. The Principle of Hash Algorithm. Image similarity detection based on the hash algorithm $[32,33]$ is frequently used in image duplicate checking [34], image denoising [35], face recognition [36], subterranean pipeline recognition [37], and other applications. The hash algorithm first turns the image data into a string and then makes a comparative analysis to determine the similarity of images [38]. We assume that the gray value of the pixels in row $i$ and column $j$ in images is $A_{i j}$ ; its average value is $A_{a}$. The hash algorithm is described below.

2.1.1. Average Hash Algorithm. The average hash algorithm (aHash) generates binary strings from low-frequency information in images to determine the similarity between images. The theory is simple, and the calculation speed is rapid, but it is easy to be disturbed by gamma correction and histogram equalization. The steps of the algorithm are as follows [34]:

(1) Preprocess the image, reduce the image size to $n \times n$, and filter the high-frequency components of images

(2) Carry out the grayscale transformation and calculate the average value $A_{a}$. The purpose is to filter the high-frequency components of images again

(3) Compare the size of $A_{i j}(i=1,2,3 \cdots n, j=1,2,3 \cdots$ $n)$ and $A_{a}$. If $A_{i j} \geq A_{a}$, the value is 1 ; otherwise, it is 0 , and $n^{2}$ binary numbers are obtained
(4) The image hash value is the string in step (3), and the Hamming distance of two strings is calculated to obtain the similarity value

2.1.2. Perceptual Hash Algorithm. Compared with the aHash, the perceptual hash algorithm (pHash) employs the discrete cosine transform (DCT), retains more image details, and is not affected by a small number of modifications in the image. Still, its computing speed is the slowest [34]. The specific steps are as follows:

(1) Preprocess the image, reduce the image size to $n \times n$, and filter the high-frequency components of the image

(2) Carry out the grayscale transformation, then DCT is performed, and the upper left corner of the transformed matrix is taken, with the size of $k \times k$. The average value $A_{a}$ is calculated

(3) Compare the size of $A_{i j}(i=1,2,3 \cdots k, j=1,2,3 \cdots$ $k$ ) and $A_{a}$. If $A_{i j} \geq A_{a}$, the value is 1 ; otherwise, it is 0 , and $k^{2}$ binary numbers are obtained

(4) The image hash value is the string in step (3), and the Hamming distance of two strings is calculated to obtain the similarity value

2.1.3. Different Hash Algorithm. The different hash algorithm (dHash) realizes image similarity detection based on morphing.

(1) Preprocess the image and reduce the image size to $(k+1) \times k$

(2) Carry out the grayscale transformation on the image of $(k+1) \times k$

(3) Compare the size of $A_{i j}(i=1,2,3 \cdots k+1, j=1,2,3$ $\cdots k-1)$ and $A_{i(j+1)}$. If $A_{i j} \geq A_{i(j+1)}$, the value is 1 ; otherwise, it is 0 , and $k^{2}$ binary numbers are obtained

(4) The image hash value is the string in step (3), and the Hamming distance of two strings is calculated to obtain the similarity value

2.2. The Retrieval Process of CT Images. Among the three hash algorithms discussed above, aHash and dHash have similar computational efficiency, but dHash has a higher recognition accuracy than aHash. pHash has the highest recognition accuracy but the lowest computational efficiency [37]. Furthermore, if the picture is slightly modified, but the general structure stays the same, the calculation results of the pHash will be unaffected. As a result, the pHash was utilized to determine the similarity of CT images and compile the MATLAB program.

Figure 1 depicts the pHash-based CT image similarity retrieval process. First, a CT image was chosen as the sample image during the initial scanning stage. Then, the CT images exported in each subsequent scanning stage were added to 


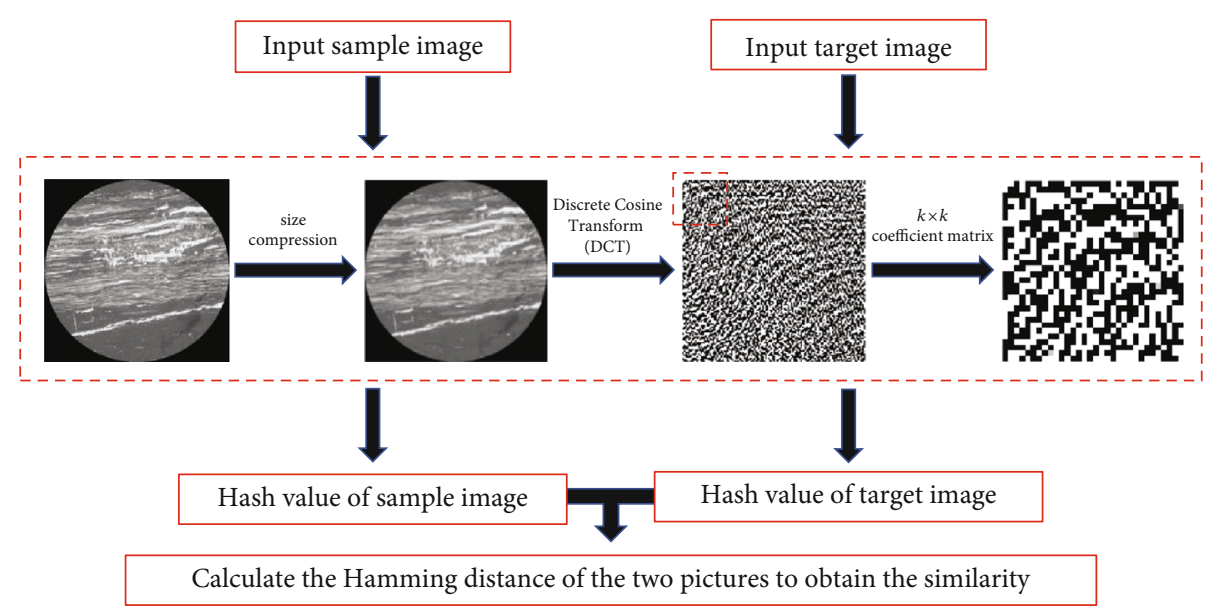

Figure 1: Similarity detection process of CT images.

the target image library. Finally, the Hamming distance was calculated between the target and sample images and performed similarity retrieval.

\section{Experiment Description}

3.1. Coal Sample Preparation. The test samples were cored from raw coal blocks from the Zhujiao coal mine in Anyang, Henan Province, China. The main mining coal seam in the Zhujiao coal mine is the No. 21 coal seam. The coal blocks were dug out from the coal mining face and sealed immediately in airtight bags. Then, the sealed coal blocks were transported to the laboratory and drilled, cut, and ground into standard cylindrical coal samples with a size of $\phi 25$ $\mathrm{mm} \times 50 \mathrm{~mm}$. The proximate analysis results and density of the coal sample are shown in Table 1.

3.2. Experiment Scheme and Procedure. Figure 2(a) depicts an industrial CT scanner that can perform 3D X-ray imaging. The industrial CT scanner was employed for the scanning experiment. The triaxial loading control system is shown in Figure 2(b), which was used to apply axial stress, confining pressure, and gas pressure on the coal sample. A carbon fiber alloy triaxial loading cell was utilized to exert confining pressure on the coal sample to minimize the effect of X-ray attenuation on the scanning accuracy.

The confining pressure in the triaxial compression test was 3.0 MPa. CT imaging quality is dependent on the scanning parameters. In this study, the parameters of scanning voltage, scanning electric current, exposure time, projection number, scanning time, and voxel size were $180 \mathrm{kV}$, $240 \mu \mathrm{A}, 2000 \mathrm{~ms}, 1500,34 \mathrm{~min}$, and $27 \mu \mathrm{m}$, respectively. The experiment steps are as follows:

(1) A coal sample was installed into the triaxial loading cell after wrapping it with a carbon fiber hose. The triaxial loading cell was then fixed on a turntable and connected to the triaxial loading control system. The axial and confining pressures were simulta- neously loaded to make the coal sample under hydrostatic pressure

(2) After a coal sample was first scanned to obtain the initial distribution of fractures, axial stress was continually applied to the coal sample. The coal sample was stopped loading and scanned six times at different deformation stages to obtain the growth and propagation of internal fractures

(3) As shown in Figure 3, several steps were executed to improve CT images clarity after all CT scans were finished. Two types of software (i.e., Phoenix Datos|x2 and VG Studio Max) were used to process and analyze the CT images to reveal the evolution of the internal fractures

3.3. The Test Result. Figure 4 shows the stress-strain curve of the coal sample and the dynamic evolution process of the three-dimensional fracture structure under external loading. The axial stresses and strains in each scanning stage of the coal sample are shown in Table 2.

The coal sample exhibited a small number of initial cracks with a volume of $10.05 \mathrm{~mm}^{3}$ (the first scan). It was gradually compacted under axial stress in the compaction stage. After entering the elastic stage, the internal cracks observed under the current scanning accuracy were fully closed (the second and third scans). The stress-strain curve was approximately a straight line. The coal sample produced irreversible plastic deformation with increased axial stress. A large number of new cracks were generated in its upper part, with a volume of $33.73 \mathrm{~mm}^{3}$, indicating that the failure of the coal sample has commenced (the fourth scan). Then, the small cracks developed, expanded, and connected to form the central fissure (the fifth scan), which promoted the instability and failure of the coal sample, with the cracks volume increasing to $98.04 \mathrm{~mm}^{3}$. After the coal sample failure, it showed the characteristics of strain softening and had a certain residual strength. The fissure structure tended to be stable, while the fissures were still developing and connected, 
Table 1: Proximate analysis and density of the coal sample.

\begin{tabular}{lcccccc}
\hline Coal rank & Moisture $\left(M_{\mathrm{ad}}\right)$ & Ash $\left(A_{\mathrm{ad}}\right)$ & Volatile matter $\left(V_{\mathrm{daf}}\right)$ & Fixed carbon $\left(F C_{\mathrm{ad}}\right)$ & True density & Pseudodensity \\
\hline Coking coal & $0.91 \%$ & $9.32 \%$ & $20.37 \%$ & $69.40 \%$ & $1.45 \mathrm{~g} / \mathrm{cm}^{3}$ & $1.33 \mathrm{~g} / \mathrm{cm}^{3}$ \\
\hline
\end{tabular}

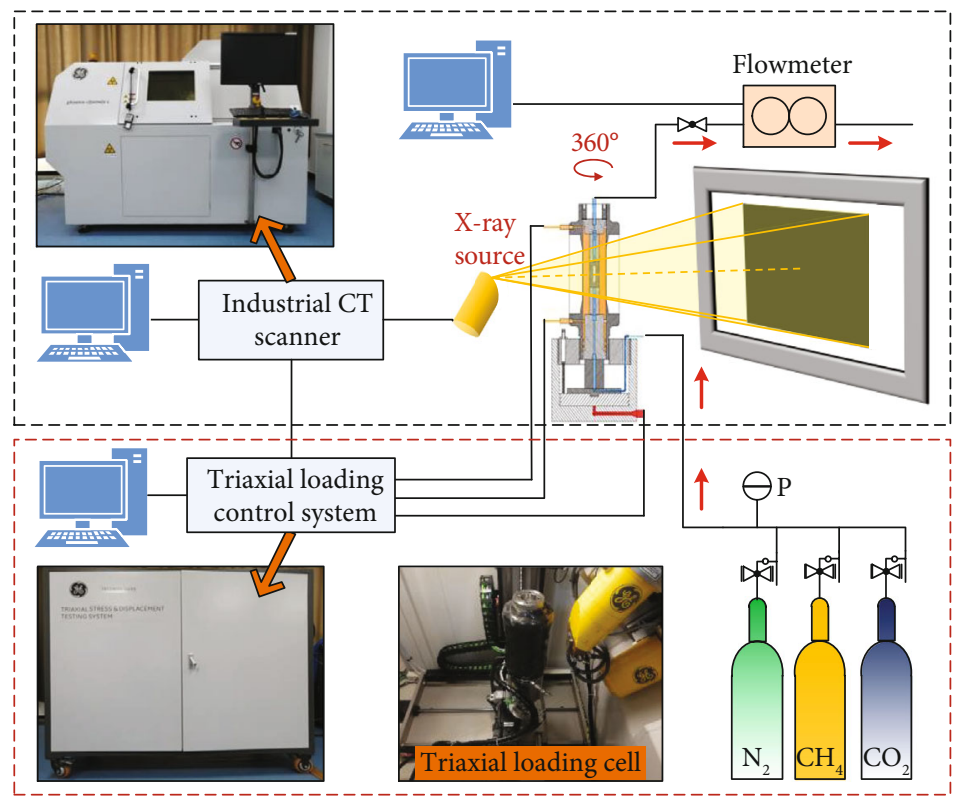
(a) The phoenix v|tome|xs industria CT scanner

FiguRE 2: Experiment apparatus.

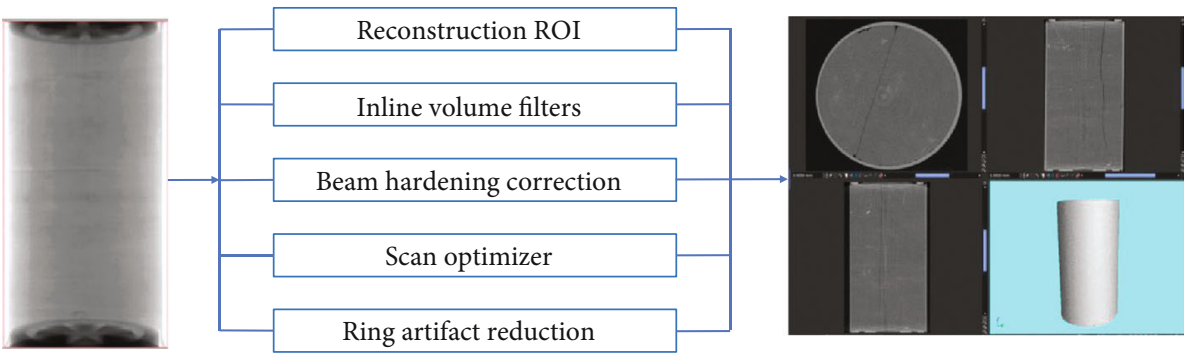

FIgURE 3: The reconstruction process of CT data.

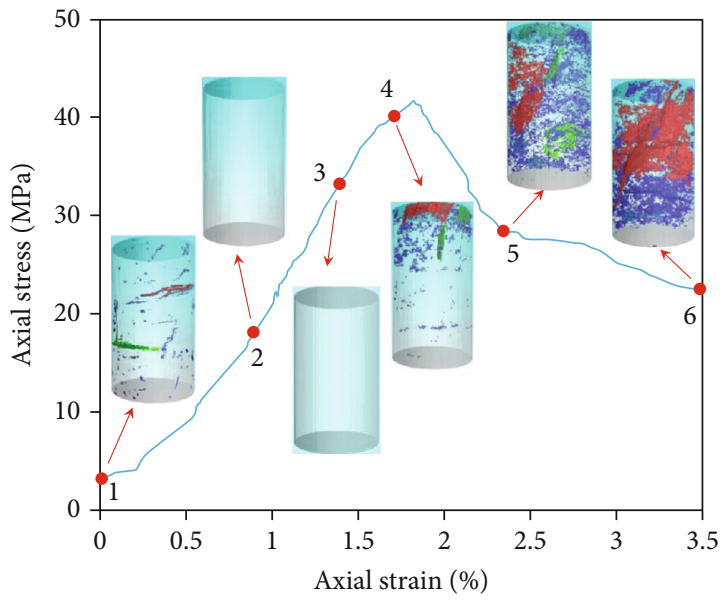

Figure 4: The stress-strain curve and three-dimensional fracture structure of the coal sample. with the volume increasing significantly to $247.15 \mathrm{~mm}^{3}$ (the sixth scan).

\section{Fracture Evolution Analysis Based on CT Similar Images}

4.1. The Similarity Retrieval of CT Images. When performing CT images similarity retrieval, each operation took the 600th layer CT image $(Z=-6 \mathrm{~mm})$ containing initial fractures in the first scan as the sample image. To avoid the influence of the end effect on the calculation results, we selected the middle area of the coal sample $(Z=18 \mathrm{~mm} \sim-18 \mathrm{~mm})$ as the ROI during CT images similarity retrieval (Figure 5). 901 CT images were exported as target images in the first to sixth scanning stages. The similarity calculation of CT images in each scanning stage of the coal sample was completed. 
TABLE 2: The scanning stage of the coal sample.

\begin{tabular}{lcccccc}
\hline The scanning stage & 1 & 2 & 3 & 4 & 5 & 6 \\
\hline Axial stress (MPa) & 3.21 & 16.55 & 33.20 & 40.23 & 28.41 & 22.51 \\
Axial strain (\%) & 0.00 & 0.85 & 1.39 & 1.72 & 2.34 & 3.49 \\
\hline
\end{tabular}

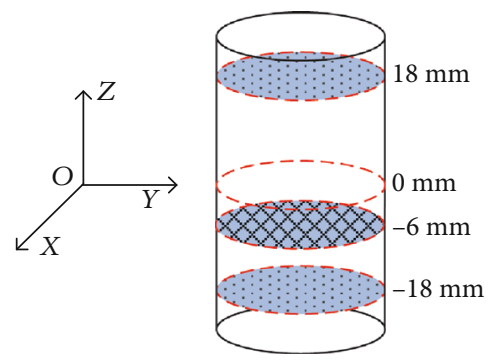

FIgUre 5: The ROI of the coal sample.

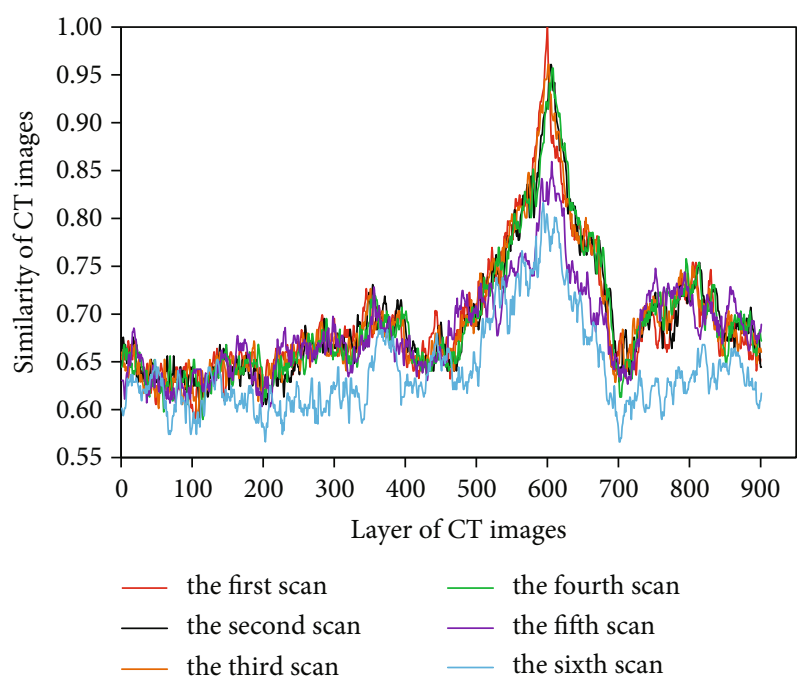

FIGURE 6: Similarity between the target and sample images in each scanning stage.

The similarity change curve of each scanning stage is shown in Figure 6, indicating the heterogeneity and anisotropy of the internal structure of the coal sample. The most similar layers are given in Table 3.

The similarity retrieval results of CT images were analyzed in combination with Figure 4. The similarity of the 600th layer image in the first scanning stage is 1 . The similarity curves in the first to fourth scanning stages are basically the same. The similarity gradually decreased with the increase of deformation of the coal sample. The most similar images in the second to fourth scanning stages are 605th, 602th, and 608th layers, respectively, with 0.960938, 0.960938 , and 0.957031 , respectively. At this time, the coal sample was in the elastic stage (the second and third scans) and plastic stage (the fourth scan), and a few primary fractures under the current scanning accuracy were closed entirely. New fractures had just begun to sprout, so the similarity changed little. During the fifth scan, the new fractures in the coal sample continued to spread and eventually pene- trate, resulting in the instability and failure of the coal sample. The most similar image is the 606th layer, and the similarity is reduced to 0.859375 . The coal sample was scanned for the sixth time in the residual strength stage, and the fractures continued to widen and connect. The most similar image is the 594th layer, and the similarity is 0.816406.

The similarity curve between the target and sample images continues to decrease after the peak point (the fifth and sixth scans) due to the continuous development and expansion of a significant number of fractures after the destruction of the coal sample. According to the similarity detection approach illustrated in Figure 1, the CT images of the coal sample in the same section under different stress states were obtained to analyze the fracture evolution more accurately.

4.2. The Similarity Quantification Analysis. The similarity of each scanning stage was quantified to characterize the evolution of mesostructure in different loading stages. Figure 7 shows the changing trend of average similarity during the deformation and failure of the coal sample. With the increase of deformation of the coal sample, the average similarity decreased steadily in the compaction, elastic, and plastic stages (the first to fourth scans), which were 0.694766 , $0.694190,0.694138$, and 0.693496 , respectively, and dropped rapidly in the postpeak stage (the fifth and sixth scans), which were 0.683381 and 0.642359 , respectively. The closure, initiation, development, and expansion of fractures led to the decline of similarity.

Assume the vector $X\left(X=\left(x_{1}, x_{2}, \cdots, x_{i}, \cdots, x_{n}\right)\right)$ represents the similarities of each CT image at the initial stage. The vector $Y\left(Y=\left(y_{1}, y_{2}, \cdots, y_{i}, \cdots, y_{n}\right)\right)$ denotes the similarities of each CT image at other scanning stages.

The absolute differences $(\delta)$ and their averages $\left(\delta_{\text {ave }}\right)$ at different scanning stages were calculated by Equations (1) and (2). In the formulas, $i$ represents the $i$ th CT crosssection image, and $n$ represents the total number of CT cross-section images. The evolution trends of the absolute differences and their averages are shown in Figure 8.

$$
\begin{gathered}
\delta=\sum_{i=1}^{n}\left(x_{i}-y_{i}\right), \\
\delta_{\text {ave }}=\frac{\delta}{n} .
\end{gathered}
$$

The subtle variation of mesostructure between the first scanning stage and other scanning stages could be found clearly. And the absolute differences of similarity could be segmented into two changing processes: a slow increase process and a rapid increase process.

In the slow increase process (i.e., 1-2, 1-3, and 1-4 stages in Figure 8), the coal sample was compressed at the compaction deformation stage, and the internal damage was gradually accumulated at the elastic deformation and plastic deformation stage, which led to the increase of the absolute differences. And in the rapid increase process (i.e., 1-5 and 1-6 stages in Figure 8), the fast generation and propagation 
TABLE 3: The similar layers in each scanning stage.

\begin{tabular}{lccccc}
\hline The scanning stage & 2 & 3 & 4 & 5 & 6 \\
\hline Similarity & 0.960938 & 0.960938 & 0.957031 & 0.859375 & 0.816406 \\
The similar layers & 605 & 602 & 608 & 606 & 594 \\
\hline
\end{tabular}

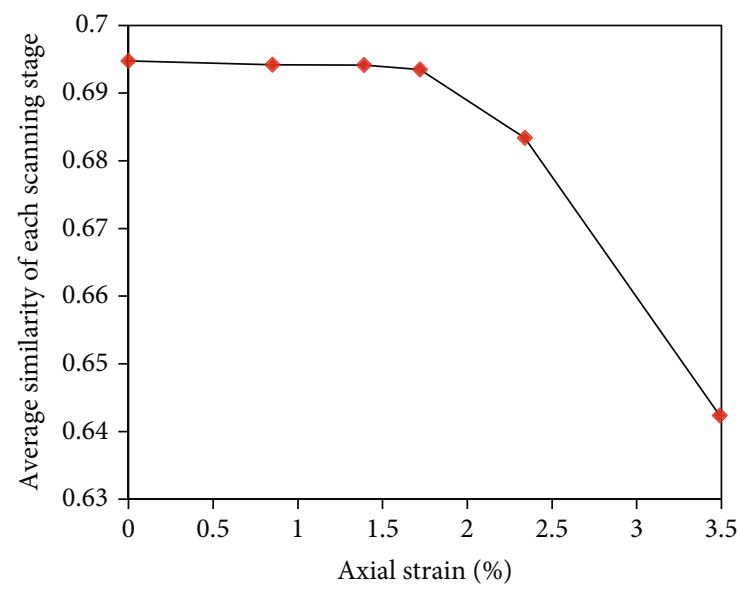

Figure 7: Average similarity in each scanning stage.

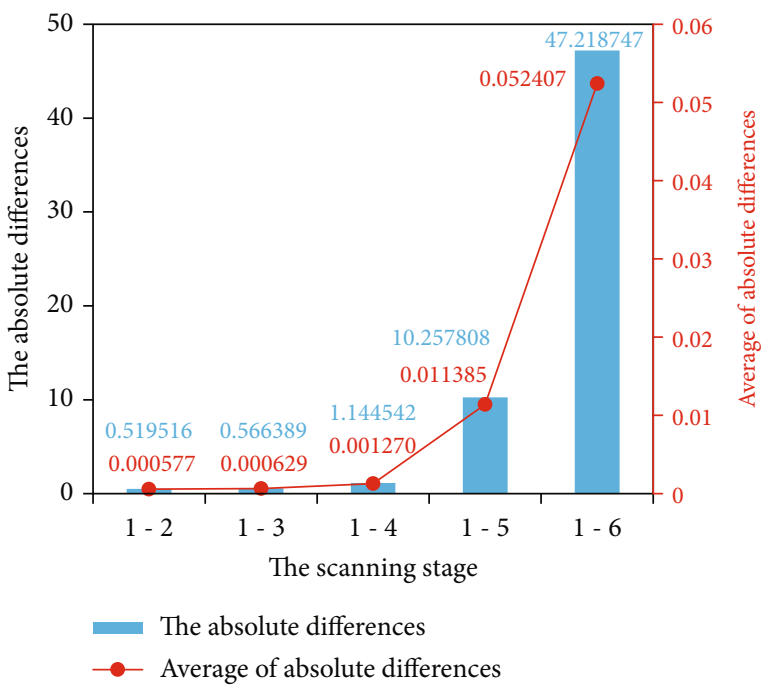

FIGURE 8: The absolute differences of similarity between the first scan and other scans.

of fractures quickly boomed the damage at the postpeak deformation stage, which then caused the dramatic increase of the absolute differences until the coal sample entered the residual strength stage. The absolute difference gradually increased from 0.519516 to 1.144542 in the slow increase process (1-2, 1-3, and 1-4 stages), and it quickly rose to 10.257808 and 47.218747 , respectively, in the rapid increase process (1-5 and 1-6 stages). It shows that the coal sample experienced a compaction process at the first stress stage, an elastic process at the second stress stage to the third stress stage, a plastic process at the fourth stress stage, and then a failure process at the fifth stress stage to the sixth stress stage.
4.3. The Analysis of CT Similar Images. The real-time information of each section in the coal sample can be obtained by industrial CT scanning, and the gray value in the CT image is positively correlated with the density of the coal sample. The high-density minerals are displayed in white, the defects such as holes and fissures are shown in black, and the coal matrix is displayed in gray [39].

The CT images of the coal sample in the same section under different stress states are located in 600th, 605th, 602th, 608th, 606th, and 594th layers, respectively, as shown in Figure 9(a). The original CT images were processed by the ternary method and $K$-means clustering algorithm. Figures 9(b) and 9(c) show the processing results of CT images, so the fracture distribution and evolution characteristics of the loaded coal sample can be analyzed.

The mineral content in the coal sample is high, forming a large number of strip mineral belts. There is a primary fracture almost penetrating the section on the CT image of the 600th layer (at the red circle in the first scan of Figure 9), which was closed in the compaction and elastic stages. The difference between the properties of minerals and coal matrixes leads to weak strength at the junction between them. Therefore, in the plastic stage, the new fractures of the coal sample first occurred at the junction and then continued to develop and expand under axial stress, which eventually led to the instability and failure of the coal sample. When the coal sample was in the residual strength stage, the fracture structure further developed, and the fracture network continued to expand. The primary fracture reopened with the further pressure relief of the coal sample (at the red circle of the sixth scan in Figure 9) and was connected with the new fractures. The location distribution of the primary fracture at the two red circles is basically the same, which explains the accuracy of the similarity retrieval of CT images to a certain extent. The above analysis is helpful to understand the failure mechanism of coal deep.

As shown from Figures 9(b) and 9(c), the coal sample contains many minerals and defects. $K$-means clustering algorithm can distinguish coal matrix and minerals. However, the segmentation effect of fractures is not as good as the ternary method, and fractures can be identified when the coal samples are fully destroyed. In this paper, we used the ternary CT images to calculate the fracture ratio at different loading stages. From the first scan to the sixth scan, the fracture ratio is $0.21 \%, 0 \%, 0 \%, 0.08 \%, 0.39 \%$, and $2.43 \%$, respectively. The variation trend of similarity, fracture ratio, and fracture volume during the stress-strain process of the coal sample was drawn, as shown in Figure 10.

In the compaction and elastic stages, the initial fractures of the coal sample were closed under external loading, and the fracture ratio of the same section was slowly reduced from $0.21 \%$ to $0 \%$. Therefore, the similarity was reduced to 


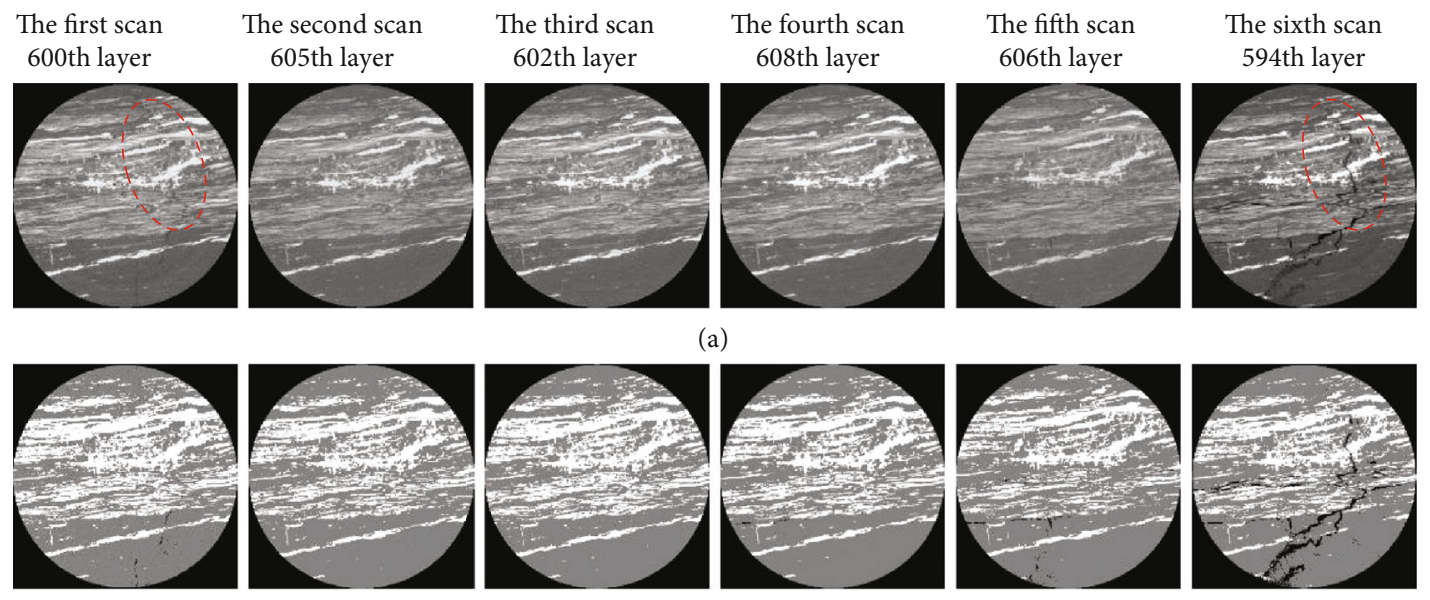

(b)
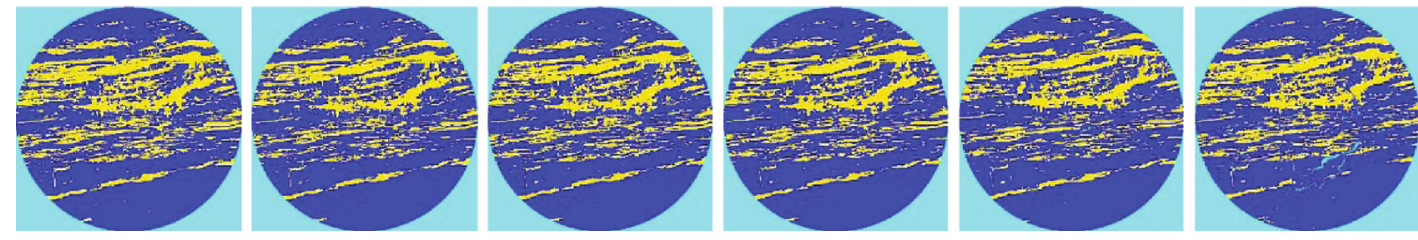

(c)

Figure 9: The processing results of CT images.

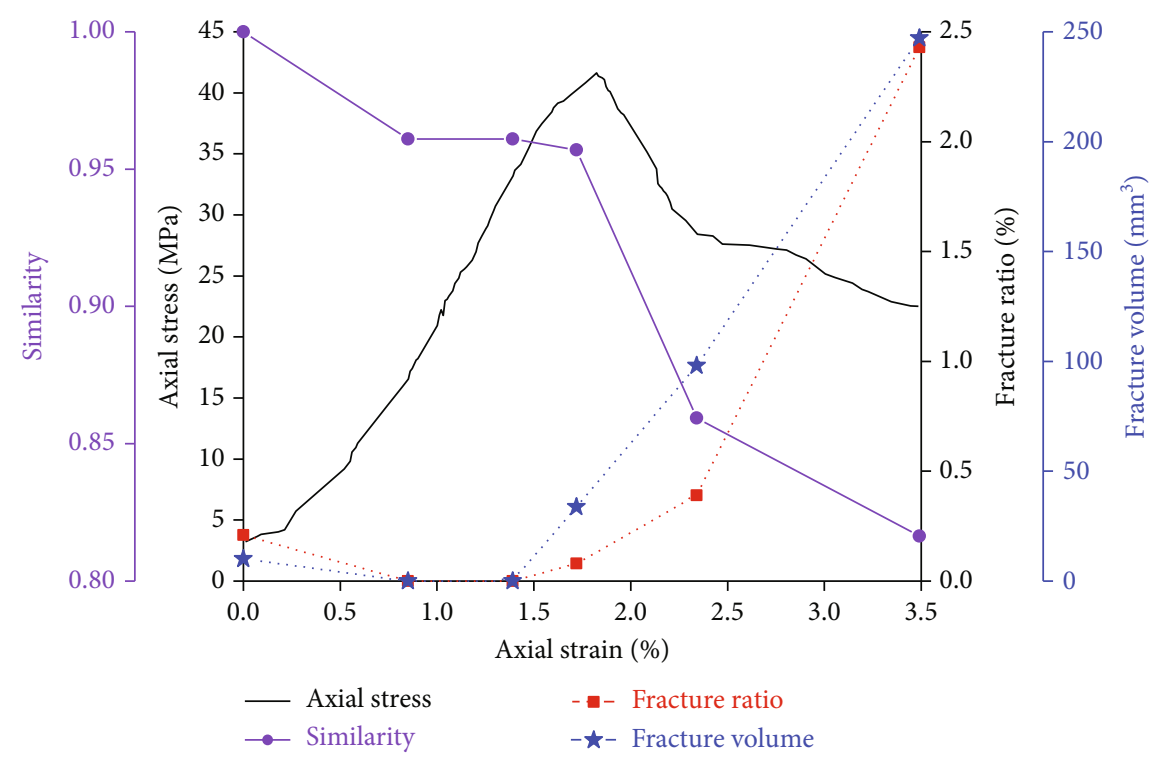

FIgUre 10: The fracture evolution trend of the coal sample.

0.960938 . New fractures steadily developed in the plastic stage, the fracture ratio grew to $0.08 \%$, and the similarity fell to 0.957031 . After the coal sample was destroyed, the fracture ratio increased sharply to $2.43 \%$, reducing the similarity to 0.816406 .

The changing trend of fracture ratio is basically the same as fracture volume. The fracture mainly experienced three stages: the closure in the compaction and elastic stage, the initiation and development in the plastic yield stage, and the rapid expansion and penetration in the postpeak stage. The overall change trend is consistent with the research results of other scholars $[27,29,31]$. It shows that the damage accumulation process of the coal sample is completed quickly in the prepeak stage near the peak point or the postpeak stage. The ternary method can be used to calculate the fracture ratio and then reflect the fracture development process and damage evolution degree of the coal sample in different deformation stages. 


\section{Conclusions}

Based on the real-time CT scanning test results of the loaded coal sample, the CT images of the coal sample in the same section under different stress states were obtained and analyzed by using image retrieval technology, and the following conclusions were drawn:

(1) Based on the perceptual hash algorithm, the similarity retrieval method of CT images was established. It can be used to identify the CT images in the same section of the coal sample under different deformation stages and more accurately analyze the mesostructure evolution in the coal sample during external loading

(2) The three-dimensional fracture structure of the loaded coal sample under different stress states was obtained by the industrial CT scanner. The closure in the compaction and elastic stage, the initiation and development in the plastic yield stage, and the rapid expansion and penetration in the postpeak stage of the internal fractures were observed, reflecting the damage evolution of the coal sample from the side

(3) For the coal containing many minerals and defects, the ternary method can better divide the coal matrix, minerals, and fractures, to calculate the fracture ratio. The overall change trend of the twodimensional fracture ratio is consistent with the fracture volume, reflecting the fracture development process and damage evolution degree of the coal sample in different deformation stages

\section{Data Availability}

The data used to support the findings of this study are available from the corresponding author upon request.

\section{Conflicts of Interest}

The authors declare that they have no conflicts of interest.

\section{Acknowledgments}

The work was supported by the Program for Guangdong Introducing Innovative and Entrepreneurial Teams (No. 2019ZT08G315), the National Natural Science Foundation of China (Nos. U2013603, 52174174, and 51774118), the Program for Leading Talents in Scientific and Technological Innovation of Henan Province (204200510032), the Key Scientific Research Projects of Universities in Henan Province (21zx004), and Shenzhen Basic Research Project (General Project) (JCYJ20190808153416970).

\section{References}

[1] M. Gao, M. Wang, and J. Xie, "In-situ disturbed mechanical behavior of deep coal rock," Journal of China Coal Society, vol. 45, no. 8, pp. 2691-2703, 2020.
[2] C. Jie, C. Zhu, D. Junsheng et al., "A quantitative pre-warning for coal burst hazard in a deep coal mine based on the spatiotemporal forecast of microseismic events," Process Safety and Environmental Protection, vol. 159, pp. 1105-1112, 2022.

[3] M. Gao, J. Xie, Y. Gao et al., "Mechanical behavior of coal under different mining rates: a case study from laboratory experiments to field testing," International Journal of Mining Science and Technology, vol. 31, no. 5, pp. 825-841, 2021.

[4] M. Gao, Z. Zhang, Y. Xiangang, C. Xu, Q. Liu, and H. Chen, "The location optimum and permeability-enhancing effect of a low-level shield rock roadway," Rock Mechanics and Rock Engineering, vol. 51, no. 9, pp. 2935-2948, 2018.

[5] C. Jiang, X. Liu, W. Wang, W. Wei, and M. Duan, “Threedimensional visualization of the evolution of pores and fractures in reservoir rocks under triaxial stress," Powder Technology, vol. 378, no. A, pp. 585-592, 2021.

[6] B. Nie, X. He, X. Li, W. Chen, and S. Hu, "Meso-structures evolution rules of coal fracture with the computerized tomography scanning method," Engineering Failure Analysis, vol. 41, no. SI, pp. 81-88, 2014.

[7] G. Mingzhong, H. Haichun, X. Shouning et al., "Discing behavior and mechanism of cores extracted from Songke-2 well at depths below 4,500 m," International Journal of Rock Mechanics and Mining Sciences, vol. 149, article 104976, 2022.

[8] M. Z. Gao, B. G. Yang, J. Xie et al., "The mechanism of microwave rock breaking and its potential application to rockbreaking technology in drilling," Petroleum Science, 2022.

[9] X. Lu, R. T. Armstrong, and P. Mostaghimi, "High-pressure Xray imaging to interpret coal permeability," Fuel, vol. 226, pp. 573-582, 2018.

[10] Y. Ju, C. Xi, Y. Zhang, L. Mao, F. Gao, and H. Xie, "Laboratory in situ CT observation of the evolution of $3 \mathrm{~d}$ fracture networks in coal subjected to confining pressures and axial compressive loads: a novel approach," Rock Mechanics and Rock Engineering, vol. 51, no. 11, pp. 3361-3375, 2018.

[11] Y. Ju, Q. Zhang, J. Zheng, J. Wang, C. Chang, and F. Gao, "Experimental study on $\mathrm{CH}_{4}$ permeability and its dependence on interior fracture networks of fractured coal under different excavation stress paths," Fuel, vol. 202, pp. 483-493, 2017.

[12] M. Gao, J. Xie, J. Guo, Y. Lu, Z. He, and C. Li, "Fractal evolution and connectivity characteristics of mining-induced crack networks in coal masses at different depths," Geomechanics and Geophysics for Geo-Energy and Geo-Resources, vol. 7, no. 1, p. 7(1), 2021.

[13] M. Gao, J.-g. Zhang, S.-w. Li, M. Wang, Y.-w. Wang, and P.f. Cui, "Calculating changes in fractal dimension of surface cracks to quantify how the dynamic loading rate affects rock failure in deep mining," Journal of Central South University, vol. 27, no. 10, pp. 3013-3024, 2020.

[14] G. Wang, J. Shen, X. Chu, C. Cao, C. Jiang, and X. Zhou, "Characterization and analysis of pores and fissures of highrank coal based on CT three-dimensional reconstruction," Journal of China Coal Society, vol. 42, no. 8, pp. 2074-2080, 2017.

[15] H. Fu, D. Tang, T. Xu et al., "Characteristics of pore structure and fractal dimension of low-rank coal: a case study of Lower Jurassic Xishanyao coal in the southern Junggar Basin, NW China," Fuel, vol. 193, pp. 254-264, 2017.

[16] G. Wang, X. Yang, X. Q. Zhang, M. Wu, and W. Li, "Numerical simulation on non-Darcy seepage of CBM by means of 3D 
reconstruction based on computed tomography," Journal of China Coal Society, vol. 41, no. 4, pp. 931-940, 2016.

[17] Y. Q. Wang, Z. C. Feng, H. Q. Guo, and D. Zhao, “CT description of rock failure process under uniaxial compression loading based on image retrieval technology," Rock and Soil Mechanics, vol. 34, no. 9, pp. 2534-2540, 2013.

[18] T. Kai and W. Zheng, "Real-time damage assessment of hydrous sandstone based on synergism of AE-CT techniques," Engineering Failure Analysis, vol. 91, pp. 465-480, 2018.

[19] Y. Zhao, Y. Sun, S. Liu, Z. Chen, and L. Yuan, "Pore structure characterization of coal by synchrotron radiation nano-CT," Fuel, vol. 215, pp. 102-110, 2018.

[20] G. Wang, J. Shen, S. Liu, C. Jiang, and X. Qin, “Three-dimensional modeling and analysis of macro-pore structure of coal using combined X-ray CT imaging and fractal theory," International Journal of Rock Mechanics and Mining Sciences, vol. 123, p. 104082, 2019.

[21] Y. Chen, J. Zuo, D. Liu, and Z. Wang, "Deformation failure characteristics of coal-rock combined body under uniaxial compression: experimental and numerical investigations," Bulletin of Engineering Geology and the Environment, vol. 78, no. 5, pp. 3449-3464, 2019.

[22] S. Tao, Z. Pan, S. Chen, and S. Tang, "Coal seam porosity and fracture heterogeneity of marcolithotypes in the Fanzhuang block, southern Qinshui Basin, China," Journal of Natural Gas Science and Engineering, vol. 66, pp. 148-158, 2019.

[23] D. Hao, S. Tu, C. Zhang, and H. Tu, "Quantitative characterization and three-dimensional reconstruction of bituminous coal fracture development under rock mechanics testing," Fuel, vol. 267, p. 117280, 2020.

[24] Y. T. Duan, X. Li, P. G. Ranjith, and Y. F. Wu, “An investigation of the evolution of the internal structures and failure modes of Longmaxi shale using novel X-ray microscopy," Journal of Petroleum Science and Engineering, vol. 184, p. 106479, 2020.

[25] D. Wang, P. Zhang, J. Wei, and C. Yu, “The seepage properties and permeability enhancement mechanism in coal under temperature shocks during unloading confining pressures," Journal of Natural Gas Science and Engineering, vol. 77, p. 103242, 2020.

[26] S. Liu, D. Wang, G. Yin, M. Li, and X. Li, "Experimental study on the microstructure evolution laws in coal seam affected by temperature impact," Rock Mechanics and Rock Engineering, vol. 53, no. 3, pp. 1359-1374, 2020.

[27] J. C. Zhong, Z. H. Wang, L. J. Wang, J. W. Zhao, W. G. Ren, and H. W. Zhou, "Characteristics of damage evolution of deep coal based on CT three-dimensional reconstruction," Journal of China Coal Society, vol. 44, no. 5, pp. 1482-1494, 2019.

[28] D. K. Wang, F. C. Zeng, J. G. Wang et al., "Dynamic evolution characteristics and fractal law of loaded coal fractures by micro industrial CT," Chinese Journal of Rock Mechanics and Engineering, vol. 39, no. 6, pp. 1165-1174, 2020.

[29] W. Tan, P. Wang, J. Wang, L. Jingjun, and W. Xue, "Mechanical properties of granite under uniaxial compression based on CT technology," Journal of Basic Science and Engineering, vol. 28, no. 6, pp. 1489-1498, 2020.

[30] L. Shuxin, X. Jie, Z. Xu, and C. Shijie, "Zonal damage information and critical failure identification of CT images of rock under triaxial compression," Chinese Journal of Geotechnical Engineering, vol. 43, no. 3, pp. 432-438, 2021.
[31] W. Dengke, W. Yan, W. Jianping et al., "Fracture dynamic evolution features of coal containing gas using gray level cooccurrence matrix and industrial CT scanning," Chinese Journal of Engineering.

[32] Z. Tang, S. Wang, X. Zhang, and W. Wei, "Structural featurebased image hashing and similarity metric for tampering detection," Fundamenta Informaticae, vol. 106, no. 1, pp. 7591, 2011.

[33] Z. Tang, S. Wang, X. Zhang, W. Wei, and Y. Zhao, "Lexicographical framework for image hashing with implementation based on DCT and NMF," Multimedia Tools and Applications, vol. 52, no. 2-3, pp. 325-345, 2011.

[34] T. A. Linchuan, D. E. Siyu, W. U. Yanxue, and W. E. Liuying, "Duplicate detection algorithm for massive images based on pHash block detection," Journal of Computer Applications, vol. 39, no. 9, pp. 2789-2794, 2019.

[35] H. Chunjian, J. Ma, and C. Ying, "Improved non-local mean denoising algorithm based on difference hash algorithm," Laser \& Optoelectronics Progress, vol. 57, no. 14, pp. 79-84, 2020.

[36] L. Deng, G. Xu, L. Mengjie, and C. ZhangJin, "Fast face recognition based on deep learning and multiple hash similarity weighting," Computer Science, vol. 47, no. 9, pp. 163-168, 2020.

[37] L. Bo, Z. Yonghui, S. Hu, S. Ruiqing, B. Wenda, and J. Weifang, "Intelligent recognition of underground pipeline from GPR image based on hash algorithm," Progress in Geophysics, 2021.

[38] C. Shuai, X. Wang, M. He, X. Ouyang, and J. Yang, "A presentation and retrieval hash scheme of images based on principal component analysis," The Visual Computer, vol. 37, no. 8, pp. 2113-2126, 2021.

[39] J. Cui, D. Liu, Y. Cai, Z. Pan, and Y. Zhou, "Insights into fractures and minerals in subbituminous and bituminous coals by FESEM-EDS and X-ray $\mu$-CT," Fuel, vol. 237, pp. 977-988, 2019. 